\title{
FY09 Advanced Instrumentation and Active Interrogation Research for Safeguards
}

D. L. Chichester

S. A. Pozzi

E. H. Seabury

J. L. Dolan

M. Flaska

J. T. Johnson

S. M. Watson

J. Wharton

August 2009

The INL is a U.S. Department of Energy National Laboratory operated by Battelle Energy Alliance

Idaho National 


\title{
FY 09 Advanced Instrumentation and Active Interrogation Research for Safeguards
}

\author{
D. L. Chichester \\ S. A. Pozzi ${ }^{1}$ \\ E. H. Seabury \\ J. L. Dolan ${ }^{1}$ \\ M. Flaska ${ }^{1}$ \\ J. T. Johnson \\ S. M. Watson \\ J. Wharton \\ ${ }^{1}$ University of Michigan
}

August 2009

Idaho National Laboratory

Idaho Falls, Idaho 83415

http://www.inl.gov

Prepared for the

U.S. Department of Energy

Office of Nuclear Energy

Under DOE Idaho Operations Office

Contract DE-AC07-05ID14517 


\section{DISCLAIMER}

This information was prepared as an account of work sponsored by an agency of the U.S. Government. Neither the U.S. Government nor any agency thereof, nor any of their employees, makes any warranty, expressed or implied, or assumes any legal liability or responsibility for the accuracy, completeness, or usefulness, of any information, apparatus, product, or process disclosed, or represents that its use would not infringe privately owned rights. References herein to any specific commercial product, process, or service by trade name, trade mark, manufacturer, or otherwise, do not necessarily constitute or imply its endorsement, recommendation, or favoring by the U.S. Government or any agency thereof. The views and opinions of authors expressed herein do not necessarily state or reflect those of the U.S. Government or any agency thereof. 


\title{
FY09 Advanced Instrumentation and Active Interrogation Research for Safeguards
}

\author{
D. L. Chichester ${ }^{1}$, S. A. Pozzi ${ }^{2}$, E. H. Seabury ${ }^{1}$, J. L. Dolan ${ }^{2}$, M. Flaska ${ }^{2}$, \\ J. T. Johnson ${ }^{1}$, S. M. Watson ${ }^{1}$, and J. Wharton ${ }^{1}$ \\ ${ }^{1}$ Idaho National Laboratory, Idaho Falls, ID 83415 \\ 2 Department of Nuclear Engineering \& Radiological Sciences, \\ University of Michigan, Ann Arbor, MI 48109
}

\begin{abstract}
Multiple small-scale projects have been undertaken to investigate advanced instrumentation solutions for safeguard measurement challenges associated with advanced fuel cycle facilities and next-generation fuel reprocessing installations. These activities are in support of the U.S. Department of Energy's Fuel Cycle Research and Development program and its Materials Protection, Accounting, and Control for Transmutation (MPACT) campaign. 1) Work was performed in a collaboration with the University of Michigan (Prof. Sara Pozzi, co-PI) to investigate the use of liquid-scintillator radiation detectors for assaying mixed-oxide (MOX) fuel, to characterize its composition and to develop advanced digital pulse-shape discrimination algorithms for performing time-correlation measurements in the MOX fuel environment. This work included both simulations and experiments and has shown that these techniques may provide a valuable approach for use within advanced safeguard measurement scenarios. 2) Work was conducted in a collaboration with Oak Ridge National Laboratory (Dr. Paul Hausladen, co-PI) to evaluate the strengths and weaknesses of the fast-neutron coded-aperture imaging technique for locating and characterizing fissile material, and as a tool for performing hold-up measurements in fissile material handling facilities. This work involved experiments at Idaho National Laboratory, using MOX fuel and uranium metal, in both passive and active interrogation configurations. A complete analysis has not yet been completed but preliminary results suggest several potential uses for the fast neutron imaging technique. 3) Work was carried out to identify measurement approaches for determining nitric acid concentration in the range of $1-4 \mathrm{M}$ and beyond. This work included laboratory measurements to investigate the suitability of prompt-gamma neutron activation analysis for this measurement and product reviews of other commercial solutions. Ultrasonic density analysis appears to be the best candidate technology for determining nitric acid concentrations but the PGNAA approach may also be applicable. 4) Work was also carried out to begin investigating the use of remote UV imaging to detect air-ionization induced by alpha particle emission from plutonium. This approach has been shown elsewhere as a useful tool for detecting and quantifying plutonium contamination and has the potential of providing a unique and powerful approach for quantifying hold-up in reprocessing facilities. Based on these simple scoping experiments the potential far-reaching capabilities of the measurement are clear.
\end{abstract}

\section{Introduction}

Advanced nuclear fuels are currently under development within the Department of Energy's Fuel Cycle Research and Development program as part of a long-term research effort focused at understanding the behavior of mixed-oxide (MOX) fuels containing minor actinides and long-lived fission products. The aim of this work is to understand how different fuel designs impact the long-term performance of nuclear fuel in order to be able to design and manufacture advanced fuels for use in next-generation 
reactors. As part of the Fuel Cycle Research and Development program's Material Protection, Accountability, and Control for Transmutation (MPACT) program Idaho National Laboratory (INL) has been performing small-scale experiments in a leveraged research program to investigate advanced instrumentation and active interrogation measurement techniques to address safeguard measurement challenges associated with advanced fuel cycle activities.

The originally allocated budget for the 2009 fiscal year (FY) was \$150,000; following the end of the continuing resolution budget stasis in the spring of 2009 an additional $\$ 50,000$ was added to the project budget, resulting in a total FY-2009 allocation of $\$ 200,000$. From these funds a subcontract was created with Prof. Sara Pozzi at the University of Michigan for $\$ 75,000$ to establish a research collaboration to perform modeling studies and experiments aimed at exploring and understanding the timecorrelated neutron emission and neutron energy spectra associated with mixed oxide (MOX) fuels. As part of that activity a week-long experimental campaign took place at INL in June to use the University of Michigan's radiation detection instrumentation to measure kg-quantity samples of MOX fuel. The total cost for planning and carrying out this campaign was roughly $\$ 60,000$; however, this MPACT project leveraged other INL funded projects dealing with neutron spectroscopy paying only approximately $\$ 40,000$ of the expenses for this effort.

In a similar fashion a second week-long experimental campaign took place at INL in July as part of a collaboration between INL and Dr. Paul Hausladen at Oak Ridge National Laboratory (ORNL). This activity explored the feasibility of using fast-neutron coded-aperture imaging techniques with passive screening and active neutron interrogation for detecting and locating fissile material, including plutonium bearing MOX fuel and enriched uranium fuel, to assess the technique as a method for locating and characterizing fissile material hold-up. The total cost for this activity was roughly $\$ 60,000$ but the cost to the MPACT program was again offset by about $\$ 20,000$ by leveraging other research activities at INL. The balance of $\$ 45,000$ was used to support two modest exploration studies, program management activities, and participation in campaign meetings. One research project examined methods for determining nitric acid concentrations in cold process streams, a topic that was identified as an important measurement need for advanced fuel cycle reprocessing facility safeguards during campaign meetings in fiscal year 2008. A second effort began to investigate the use of ultraviolet (UV) imaging techniques for detecting and quantifying alpha-emitting radioisotopes for contamination monitoring and hold-up safeguards evaluations.

\section{MOX Fuel Modeling, Simulations, \& Experiments (with the University of Michigan)}

For the 2009 fiscal year INL created a collaboration with researchers at the University of Michigan to study numerical modeling techniques for passively assaying time-correlated neutron and photon signatures from MOX fuel. Two reports were generated from this work during the current fiscal year, one dealing with preliminary modeling of the neutron emissions from ideal $10 \times 10$ element fuel assemblies, the second providing an overview of the June experimental campaign with preliminary results from those experiments.[1,2]

These analyses used the MCNP-PoliMi Monte Carlo simulation tool to determine the relative strength and energy spectra of the different neutron source terms within 
different MOX fuel blends, and then used this data to simulate the detection and measurement of these emissions using an array of liquid scintillator neutron spectrometers.[3] These calculations accounted for neutrons generated from the spontaneous fission of the actinides in the MOX fuel as well as neutrons created via $(\alpha, n)$ reactions with oxygen in the MOX fuel. The analysis was carried out to allow for characterization of both neutron energy as well as neutron coincidences between multiple detectors. Coincidences between prompt gamma rays and neutrons were also analyzed. Experiments were performed at INL's Zero-Power Physics Reactor (ZPPR) facility with the same materials used in the simulations to benchmark and begin validation tests of the simulations.[4,5] Data was collected in these experiments using an array of four liquid scintillators and a high-speed waveform digitizer. Advanced digital pulse-shape discrimination algorithms were developed and used to collect this data.

Results of the simulation and modeling studies are presented in references 1 and 2 together with preliminary results from the experimental campaign. The simulation results indicate low-resolution neutron spectroscopy may be useful for verifying the matrix form of neutron emitting materials such as MOX fuel while higher resolution neutron spectroscopy may provide a tool for determining or verifying material mass and geometry, under certain conditions. Preliminary analysis of the experimental validation data has shown reasonable agreement but has also highlighted the need to incorporate additional phenomena into the models.

The experimental campaign has also provided insight into some important challenges that need to be addressed for future activities. In the next year of the project work will continue to develop and strengthen our scientific understanding of these phenomena, and prepare for more complicated modeling using representative advanced MOX fuel compositions, spent fuel, and other advanced fuel forms. In addition, the project will expand to begin simulations and experimental analyses in active interrogation measurements. Active interrogation can be used to increase the intensity and number of signatures from special nuclear material.

\section{Fast-Neutron Coded-Aperture Imaging: Passive Screening and Active Interrogation of Fissionable Materials (with Oak Ridge National Laboratory)}

In July of 2009 a second experimental campaign was carried out at INL's ZPPR facility using some of the same materials used with the University of Michigan team, as well as a special-geometry container filled with MOX fuel pins and a container filled with approximately $9 \mathrm{~kg}$ of highly enriched uranium (HEU). These experiments were focused on evaluating ORNL's newly developed fast-neutron coded-aperture imaging system and a) assessing its passive capabilities for detecting and locating materials spontaneously emitting neutrons (plutonium or MOX fuel) for applications such as holdup determination and b) assessing its capability to be operated in an active interrogation mode for detecting and characterizing uranium. A full report is being prepared by Paul Hausladen and Matthew Blackston on this subject and will be ready from ORNL by the end of the fiscal year. ${ }^{1}$

As a demonstration of the technique preliminary data from the ORNL team is shown in Figure 1. The system resolution is roughly $2 \mathrm{~cm}$, which allows for the general outline of the material shape to be identified, rectangular versus circular, for the two

\footnotetext{
${ }^{1}$ The preliminary data presented in this section were provided by the ORNL team.
} 
orientations of the material. Typical data collection times varied from 20 minutes to a few hours, depending upon the mass of material being imaged and its stand-off distance. In a separate experiment nine of the same fuel pins were arranged in a pattern forming the letter "I" within a fuel can and then passively imaged; these results are shown in Figure 2. One further example of the system's imaging capabilities is shown in Figure 3, where the same can of MOX fuel pins from Figure 1 has been placed behind a brick wall 5 meters from a slightly larger coded aperture. Here the high intrinsic radiation emission rate from the MOX fuel makes it clearly visible behind the wall. Although only preliminary analyses were ready at the time of writing this report the utility and benefits of using advanced imaging systems for nonproliferation measurements and inspections appears quite strong. Future work in this are must examine the relative contribution of neutrons versus gamma rays in the ORNL imaging system, and may suggest the need to use a particle-specific radiation sensor such as a liquid scintillator for the imaging screen, rather than the plastic scintillators used for these measurements.
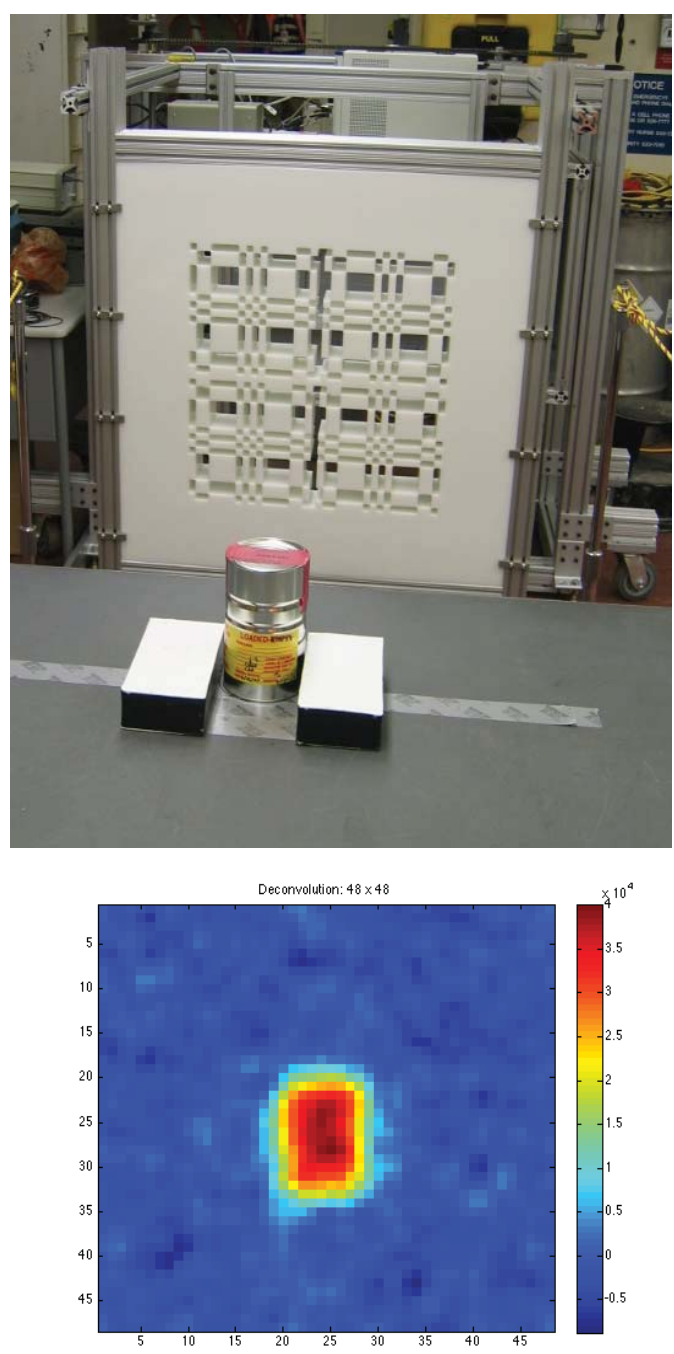
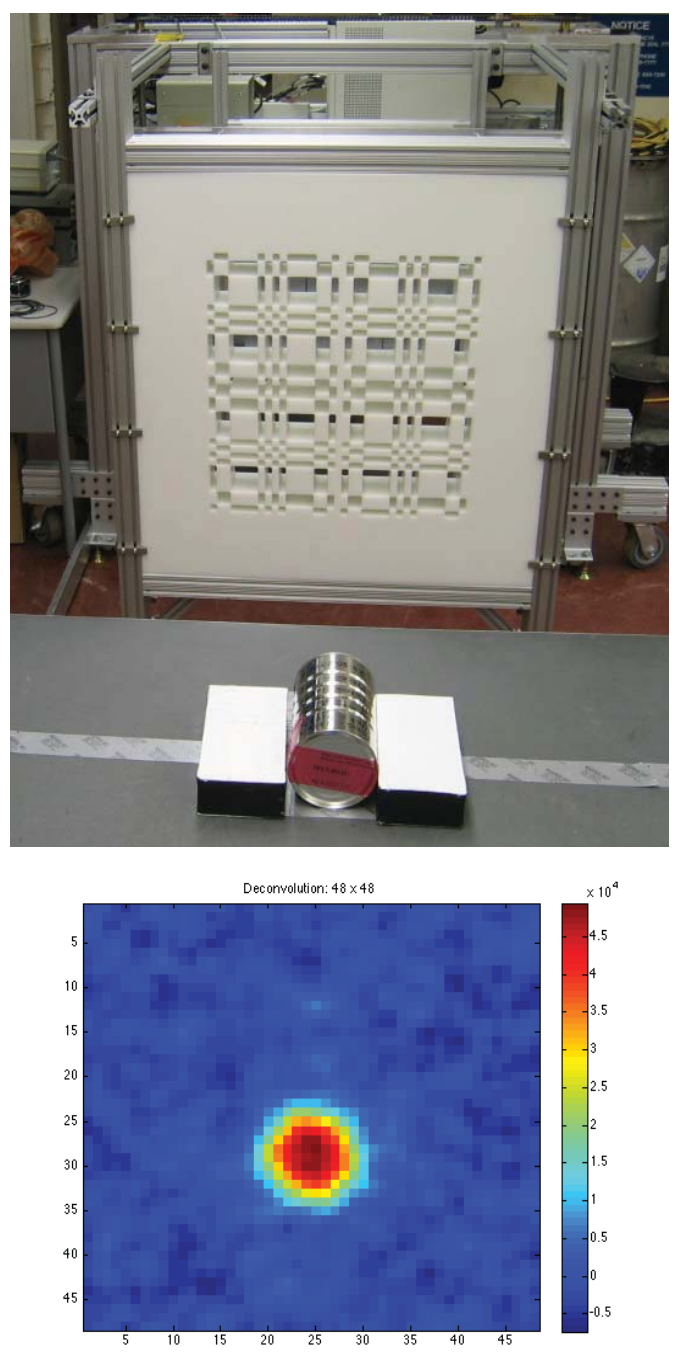

Figure 1 The experimental setup (top) and fast-neutron coded-aperture images (bottom) taken of the spontaneous neutron sources from a storage can filled with $90 \mathrm{MOX}$ fuel pins in an upright (left) and horizontal (right) orientation. 

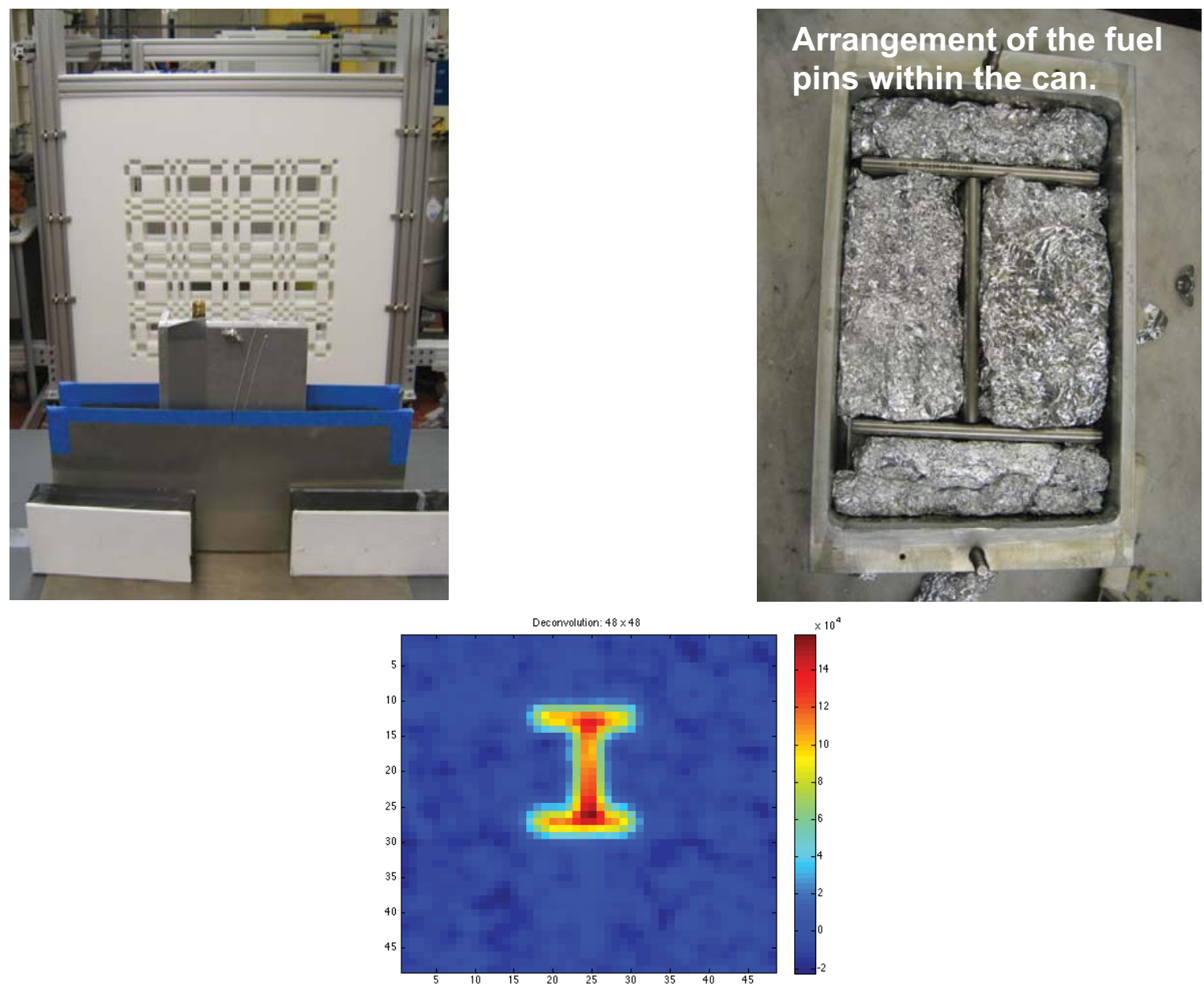

Figure 2 Experimental setup (top left) used to image a storage can filled with nine MOX fuel pins oriented in an "I" layout (top right) and preliminary data of the reconstructed coded-aperture image (bottom).
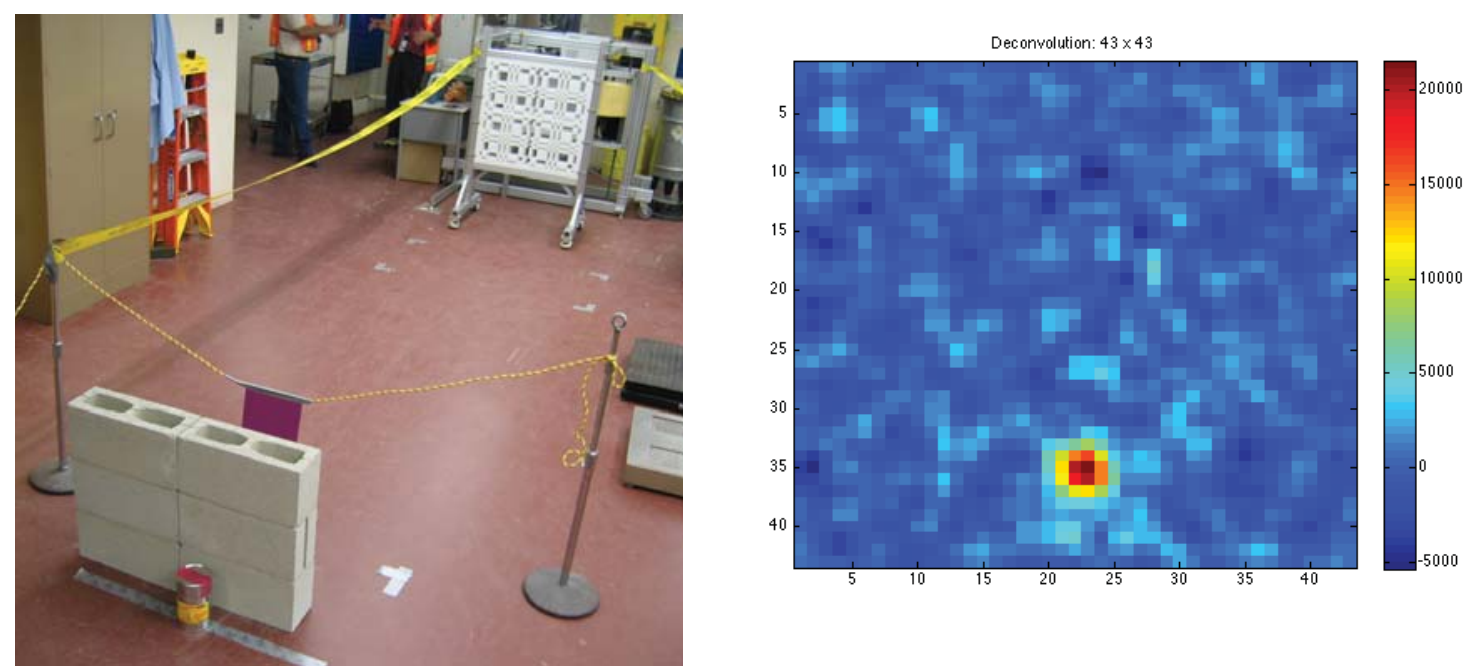

Figure 3 Experimental setup and data for imaging MOX fuel behind a brick wall at a distance of 5 meters. 


\section{Review of On-Line Monitoring Techniques to Determine Cold-Stream Nitric Acid Strength}

During a 2008 Safeguards Campaign quarterly meeting Argonne National Laboratory presented information suggesting that there would be an important need to be able to measure the concentration of the cold-stream $\mathrm{HNO}_{3}$ feed entering some advanced nuclear fuel reprocessing systems under consideration in the Advanced Fuel Cycle Initiative.[6] Further, at that meeting the general subject of monitoring the cold-stream products in advanced fuel reprocessing facilities was discussed and described as an important topic. Based on these discussion INL chose to examine potential solutions for addressing this subject; these findings are presented in this section. In retrospect recent programmatic shifts within the Fuel Cycle Research and Development program, where increasing emphasis is being placed on basic scientific exploration and less emphasis is being placed on engineering analyses, appears to have diminished the need for addressing this subject and its importance is no longer as high as it was last year. In particular, programmatic changes related to construction timelines for the advanced fuel cycle facility have diminished urgency for a solution. Nevertheless, some work has been done to evaluate solutions for addressing this process measurement requirement and these findings are presented here.

\section{Prompt-Gamma Neutron Activation Analysis}

Strong nitric acid $(>1 \mathrm{M})$ is used in many industrial chemical processes and the need to accurately measure nitric acid in this concentration range is often important in these applications. However, current techniques to perform this measurement involve the use of sensors that make contact with the acid directly. Some of these sensors require periodic maintenance, which typically requires the pipe sections where they are installed to be shut off and emptied. This requirement is tolerated in plant processes operated in batch mode but it is a significant burden at plants which would otherwise operate in continuous operating modes for long periods of time, or in facilities where personnel access is limited for security or safeguards reasons. For nuclear fuel reprocessing, safety and quality procedures associated with accessing a process stream add complexity to the steps needed for opening a pipe section to perform maintenance on a nitric acid sensor. One possible solution is to use prompt-gamma neutron activation analysis (PGNAA) to measure the nitrogen content of a test volume (a pipe) filled with nitric acid and to use that measurement to infer nitric acid concentration. High strength nitric acid in the range of $0.25 \mathrm{M}$ to $5 \mathrm{M}$ contains up to 70 grams of nitrogen per liter of solution, as presented in Table 1. To investigate the PGNAA technique in this application our research evaluated the feasibility of adapting INL's PINS (portable isotopic neutron spectroscopy) system for assaying the nitrogen concentration of nitric acid.[7] This was a logical step for our research group since the PINS system is routinely used to perform prompt gamma-ray neutron activation analysis (PGNAA) measurements of objects suspected of containing nitrogen bearing materials, including chemical warfare materiel and high-explosives. 
Table 1 Parameters for nitrogen in nitric acid

\begin{tabular}{|c|c|c|c|c|c|c|}
\hline \multirow{2}{*}{ Parameter } & \multicolumn{5}{|c|}{ Nitric Acid Molarity } \\
\hline $\begin{array}{c}\text { Nitric acid in solution } \\
\text { (wt. \%) }\end{array}$ & $\mathbf{0 . 2 5}$ & $\mathbf{0 . 5}$ & $\mathbf{1}$ & $\mathbf{2}$ & $\mathbf{4}$ & $\mathbf{5}$ \\
\hline $\begin{array}{c}\text { Nitrogen in solution } \\
\text { (wt. \%) }\end{array}$ & 0.35 & 0.69 & 1.36 & 2.63 & 4.96 & 6.03 \\
\hline $\begin{array}{c}\text { Nitrogen in solution } \\
\text { (g/L) }\end{array}$ & 3.50 & 7.00 & 14.0 & 28.0 & 56.0 & 70.0 \\
\hline
\end{tabular}

In particular, PINS is frequently used to analyze the nerve agent VX; in our laboratory we have canisters (mock $155 \mathrm{~mm}$ artillery shells) filled with a simulated mixture of VX. To evaluate the PGNAA method for nitric acid analysis measurements were first carried out using $1.5 \mathrm{~kg}$ of a mixture of polyethylene, ammonium phosphate monobasic, sulfur, and red phosphorous. This mixture is designed to simulate the nerve agent VX but has a similar concentration of nitrogen (2.7\% by weight) as $2 \mathrm{M}$ nitric acid $(2.6 \%)$. The hydrogen concentration of the simulant $(9.7 \%)$ is also similar to that of $2 \mathrm{M}$ nitric acid $(10 \%)$. The similarities in hydrogen and nitrogen concentration should lead to very similar responses between the simulant and $2 \mathrm{M}$ nitric acid, as nitrogen is detected through thermal neutron capture and subsequent gamma ray emission. Other elements in the simulant also absorb thermal neutrons, however, which does alter the degree of similarity between the simulant and that which would be expected with actual nitric acid.

Separate measurements were taken using a $\mathrm{LaBr}$ scintillator, a $\mathrm{NaI}$ scintillator, and a high purity germanium detector (HPGe) solid-state detector. (For a description of the typical PINS setup see reference 7.) The small size of this simulant limited our ability to collect an acceptable nitrogen signature over $0.28-0.83$ hour acquisition runs. Indeed, no discernable signal was observed using the $7.62 \mathrm{~cm} \times 7.62 \mathrm{~cm}$ diameter NaI or $\mathrm{LaBr}$ detectors in our non-optimized setup, while only a small signal was observed with the HPGe detector. Settling on using the HPGe detector because of its superior spectral resolution, two more measurements were taken with the small VX simulant, one for 1.4 hours and the other for 4.2 hours. Both of these second-round measurements used a polyethylene annulus around the test objects to increase neutron thermalization and the associated thermal neutron capture gamma ray signature from nitrogen. The first measurement yielded a total of $91 \pm 19$ counts in the nitrogen peaks and the second yielded $288 \pm 31$ counts. A plot of the nitrogen region of the 4.2 hour spectrum is shown in Figure 4. Using these values as a basis, we estimate a non-optimized PGNAA measurement using the PINS system could discriminate between $4 \mathrm{M}$ nitric acid and $2 \mathrm{M}$ nitric acid to $95 \%$ confidence with count times approaching 1.8 hours.

The dead time for these measurements was 38\%; this could likely be improved with enhanced shielding but it is not likely to be reduced below approximately the $20 \%$ level while still maintaining an adequate view of the sample. This would correspond to a real counting time of 1.4 hours to discriminate between $2 \mathrm{M}$ and $4 \mathrm{M}$ nitric acid. 
Discrimination between lower concentrations of acid would take longer while discrimination between a very low and a very high concentration would be quicker.

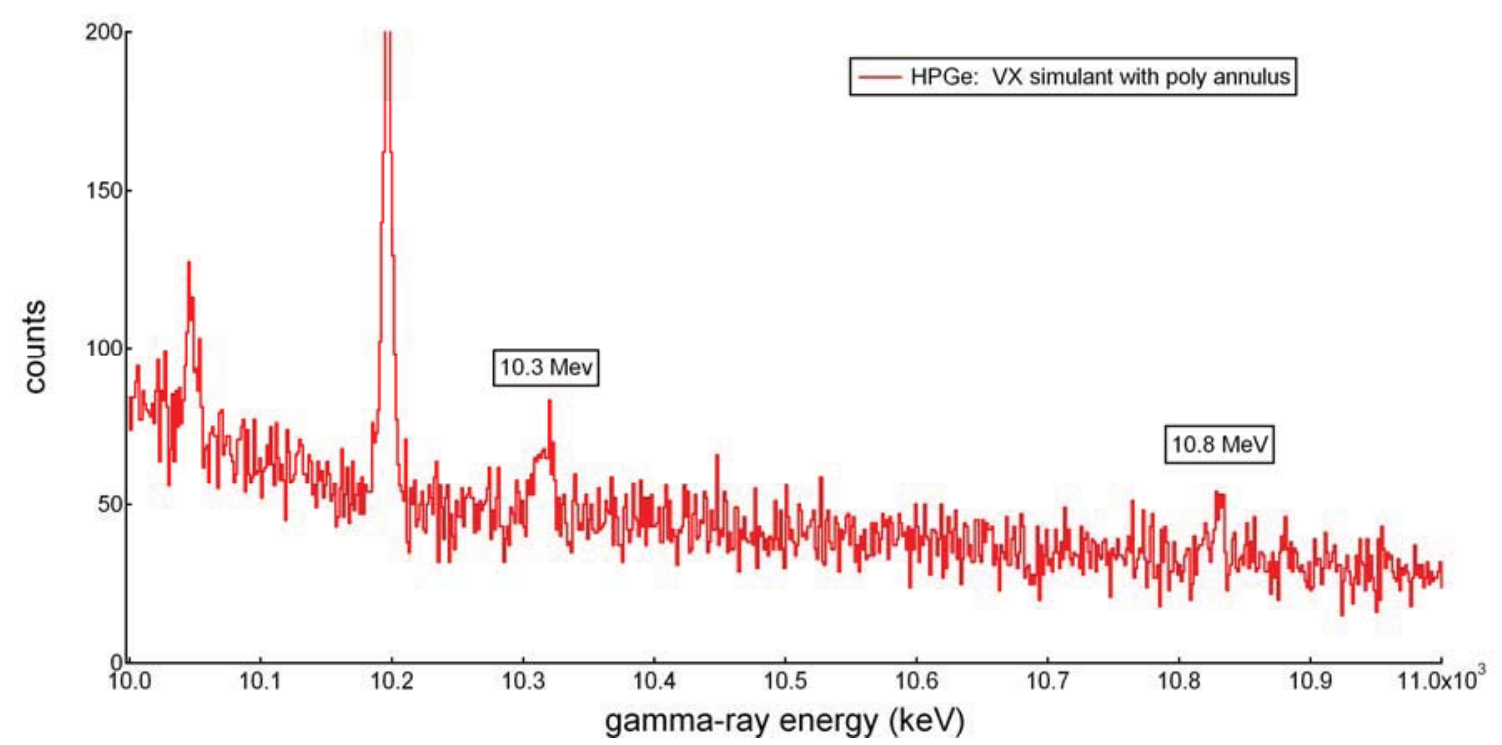

Figure 4 High-energy PGNAA spectra of the VX simulant taken with an HPGe detector for 4.2 hours using 10 micrograms of ${ }^{252} \mathrm{Cf}$.

Recognizing that this first approach was not optimized a second set of experiments was carried out using a larger sample of nitrogen-bearing material and a polyethylene reflecting box. The enclosure consisted of six $5.08-\mathrm{cm}$ thick pieces of polyethylene arranged in a hexagonal pattern to make the walls and a single large $2.5-\mathrm{cm}$ thick cap. Each wall is $61-\mathrm{cm}$ tall and $31.7-\mathrm{cm}$ wide, with additional $6.4-\mathrm{cm}$ diameter hinges on each side joint. The walls were interconnected through the use of a $66-\mathrm{cm}$ long polyethylene pins at each of the six connecting joints. The removal of one hinge-pin allowed access to the enclosure for the introduction and change-out of experimental samples. The base of the enclosure was made of aluminum and stands on three $89-\mathrm{cm}$ long aluminum legs. A photo of the irradiation box is shown in Figure 5.

The neutron source used for these measurements was $3.88 \mu \mathrm{g}(2.1 \mathrm{mCi})$ of ${ }^{252} \mathrm{Cf}$, producing approximately 9 million neutrons per second. It was placed in a cube of polyethylene, $10-\mathrm{cm}$ long per side; the source's placement in the cube is shown in the inset of Figure 5. The cube containing the ${ }^{252} \mathrm{Cf}$ source was stacked on top of other pieces of polyethylene until its center-line height was $30.5-\mathrm{cm}$ above the aluminum enclosure base, placing it in the vertical center of the enclosure. The height of the centerline of the experimental sample was adjusted to be in the vertical center of the box by placing it on a lab jack and adjusting the height. The detector used for these measurements was an HPGe detector (relative efficiency at $1.33 \mathrm{MeV}$ of $47.7 \%$ ). For these experiments three different experimental samples were chosen. A sample with very high nitrogen content was used to give a "best case" scenario for nitrogen detection; this was a 3-gallon bucket of high explosive (HE) simulant. A VX simulant was chosen because its nitrogen content closely matches that of $2 \mathrm{M}$ nitric acid. A GB simulant was used to verify any nitrogen seen in the VX spectra was real and not from the liquid nitrogen Dewar of the detector (GB has a similar chemical composition to VX but does 
not contain nitrogen.) Both the VX and GB simulants are replicates of 155-mm artillery projectiles that contain approximately $2.3 \mathrm{~kg}$ of fill materials. The nitrogen and hydrogen contents of each sample are listed in Table 2.

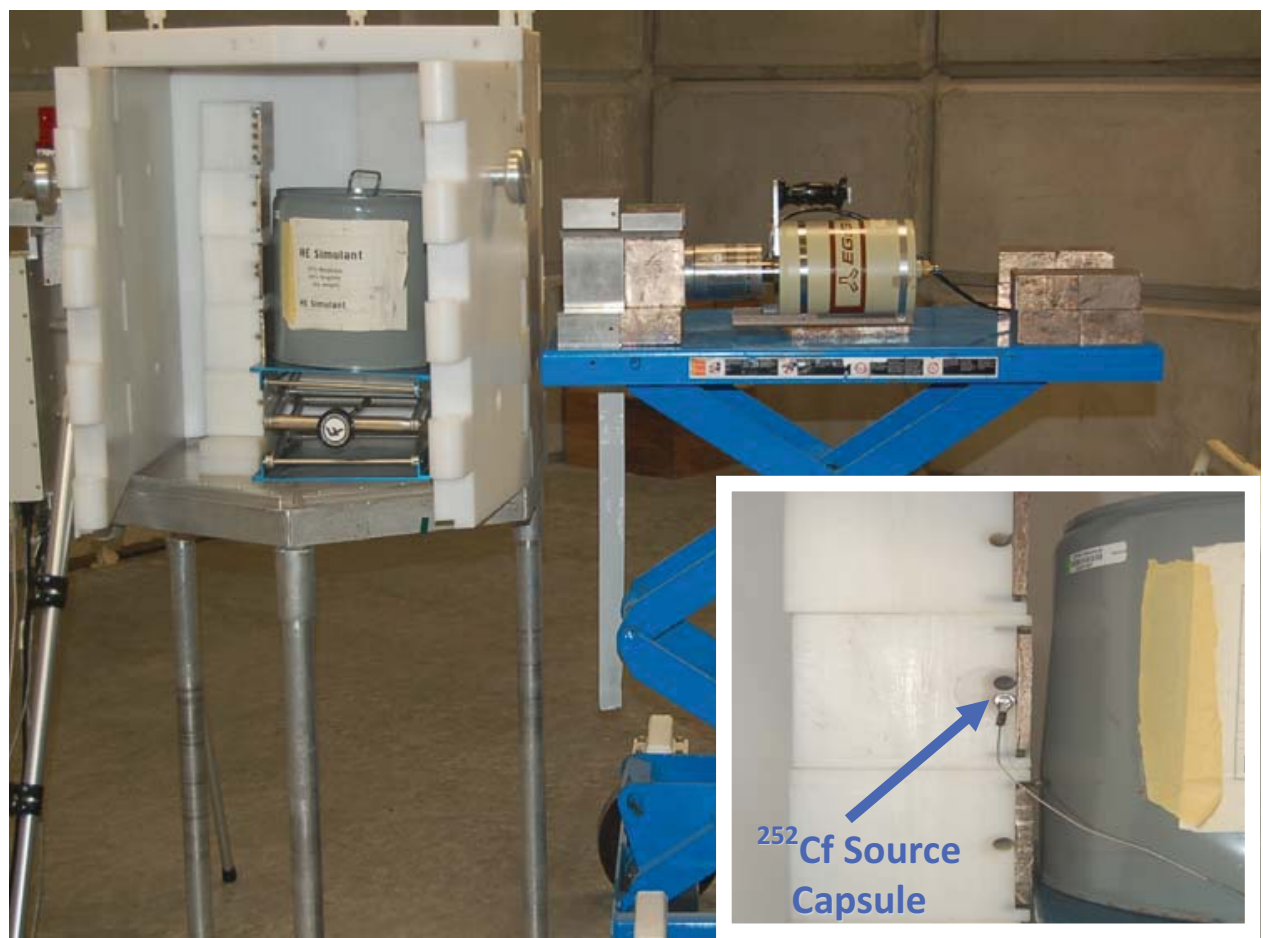

Figure 5 Photograph of the experimental set-up used in the second set of PGNAA measurements, showing the HPGe detector in a bismuth-tungsten collimator pointing at the box, with a 3-gallon bucket of high-explosive simulant inside the box. In the insert an arrow points to the location of the ${ }^{252} \mathrm{Cf}$ source.

Table 2 Nitrogen and hydrogen composition of the VX, GB, and HE simulants

\begin{tabular}{|c|c|c|}
\hline Item & Nitrogen (weight \%) & Hydrogen (weight \%) \\
\hline VX simulant & 2.74 & 9.76 \\
\hline GB simulant & 0.00 & 8.09 \\
\hline HE simulant & 38.0 & 2.74 \\
\hline
\end{tabular}

For the first set of the experiments, the experimental HE sample was placed in the center of the enclosure. The neutron source was placed in the center polyethylene block and the "doors" were closed on the enclosure. This was performed for only the HE sample and for a background measurement. During the background measurement, a small piece of tungsten was placed in front of the source to reduce the detector dead time. The GB and VX samples were measured with the source and experimental sample shifted closer to the enclosure wall nearest the detector. Two sets of measurements were 
performed with these samples in this configuration. During the first set, the source was positioned facing the sample as before. During the second setup, the source block was turned $180^{\circ}$, placing 4 inches of polyethylene between the source and the sample, increasing neutron moderation, a photograph of this arrangement is shown in Figure 6.

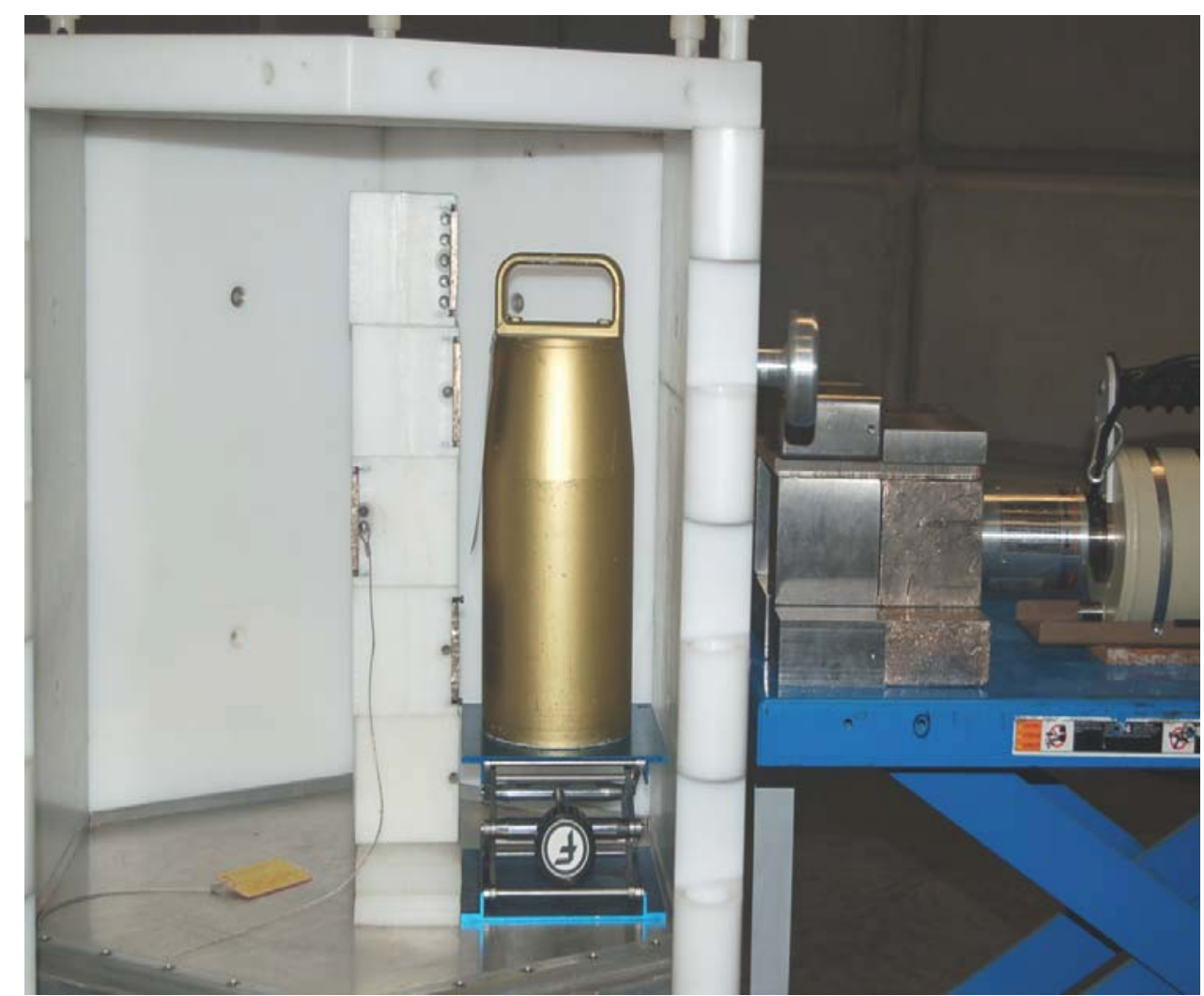

Figure 6 Experimental setup for analyzing the 155-mm artillery shell in the poly box with the ${ }^{252} \mathrm{Cf}$ source rotated $180^{\circ}$

Table 3 PINS PGNAA results for test items inside the box

\begin{tabular}{|c|c|c|c|}
\hline $\begin{array}{c}\text { Sample } \\
\text { Description }\end{array}$ & $\begin{array}{c}\text { Hydrogen at } \\
\mathbf{2 , 2 2 3} \mathbf{k e V} \\
\text { Peak Intensity } \\
\text { (counts) }\end{array}$ & $\begin{array}{c}\text { Nitrogen at } \\
\mathbf{1 0 , 3 1 8 ~ k e V} \\
\text { Peak Intensity } \\
\text { (counts) }\end{array}$ & $\begin{array}{c}\text { Nitrogen at } \\
\mathbf{1 0 , 8 2 9} \mathbf{k e V} \\
\text { Peak Intensity } \\
\text { (counts) }\end{array}$ \\
\hline Background & $\begin{array}{c}243599.65 \pm \\
501.26\end{array}$ & $1.85 \pm 2.24$ & $-0.47 \pm 1.41$ \\
\hline 3-gal bucket HE simulant & $\begin{array}{c}203146.47 \pm \\
461.06\end{array}$ & $106.59 \pm 11.05$ & $80.85 \pm 9.17$ \\
\hline $\begin{array}{c}\text { VX 155-mm } \\
\text { (source on far side) }\end{array}$ & $\begin{array}{c}227605.64 \pm \\
489.13\end{array}$ & $2.95 \pm 2.65$ & $2.30 \pm 2.24$ \\
\hline $\begin{array}{c}\text { GB 155-mm } \\
\text { (source on far side) }\end{array}$ & $\begin{array}{c}148368.40 \pm \\
396.57\end{array}$ & $2.96 \pm 2.45$ & $0.75 \pm 1.73$ \\
\hline $\begin{array}{c}\text { VX 155-mm } \\
\text { (source on near side) }\end{array}$ & $\begin{array}{c}255001.12 \pm \\
525.05\end{array}$ & $2.69 \pm 3.16$ & $6.59 \pm 3.61$ \\
\hline
\end{tabular}


Looking at the results of the measurements taken using the HPGe detector with items in the box (Table 3), no positive nitrogen was detected in either the $155-\mathrm{mm} \mathrm{VX}$ or GB. Very large peak areas were seen for both nitrogen peaks when using the 3-gallon bucket of HE simulant. These results imply that for PGNAA measurements to be performed for monitoring nitric acid strength the interrogation volume will need to be larger than that available with access to stand-alone fluid piping. For example, if a plenum area is included within a pipe section than these techniques would be more likely to prove satisfactory.

Referencing industrial process monitoring instrumentation a good example of a large-scale solution is available for comparison.[8] In this approach an instrument measuring roughly $2 \mathrm{~m} \times 2 \mathrm{~m} \times 2 \mathrm{~m}$, with a 24 microgram californium source in an optimized moderator/reflector assembly, was used to interrogate a fluid-filled volume measuring $24 \mathrm{~cm} \times 24 \mathrm{~cm} \times 34 \mathrm{~cm}(19.5 \mathrm{~L})$. A photo of the instrument is shown in Figure 7. Measurements were made using a large $15.25 \mathrm{~cm}$ long by $15.25 \mathrm{~cm}$ diameter $\mathrm{NaI}$ detector for one hour. This paper shows that a liquid solution with $2.7 \mathrm{wt} \%$ nitrogen content could be analyzed to determine the nitrogen concentration of a solution to a precision better than $3 \%$ in less than 3 minutes. An example of a one-hour spectra from the instrument is shown in Figure 8. Clearly, PGNAA can be used to provide a comprehensive and accurate solution for assaying the nitrogen content of nitric acid. However, the level of complexity for such a system may be higher than associated with typical nondestructive assay (NDA) based safeguards systems. We would estimate the cost for such a system to be approximately $\$ 300,000$, not including non-recurring engineering costs associated with its design and prototype testing. The installation footprint for this type of solution is also large compared to other NDA equipment. Equipment this size may be feasible within next generation reprocessing facilities following the Safeguards by Design approach but might be a burden on the engineering design and cost analysis of the facility. Further engineering analyses would be needed to assess these impacts versus the safeguard benefits of the measurement, including the provision of near real-time analyses and the lack of any need to penetrate or impact the process stream. 


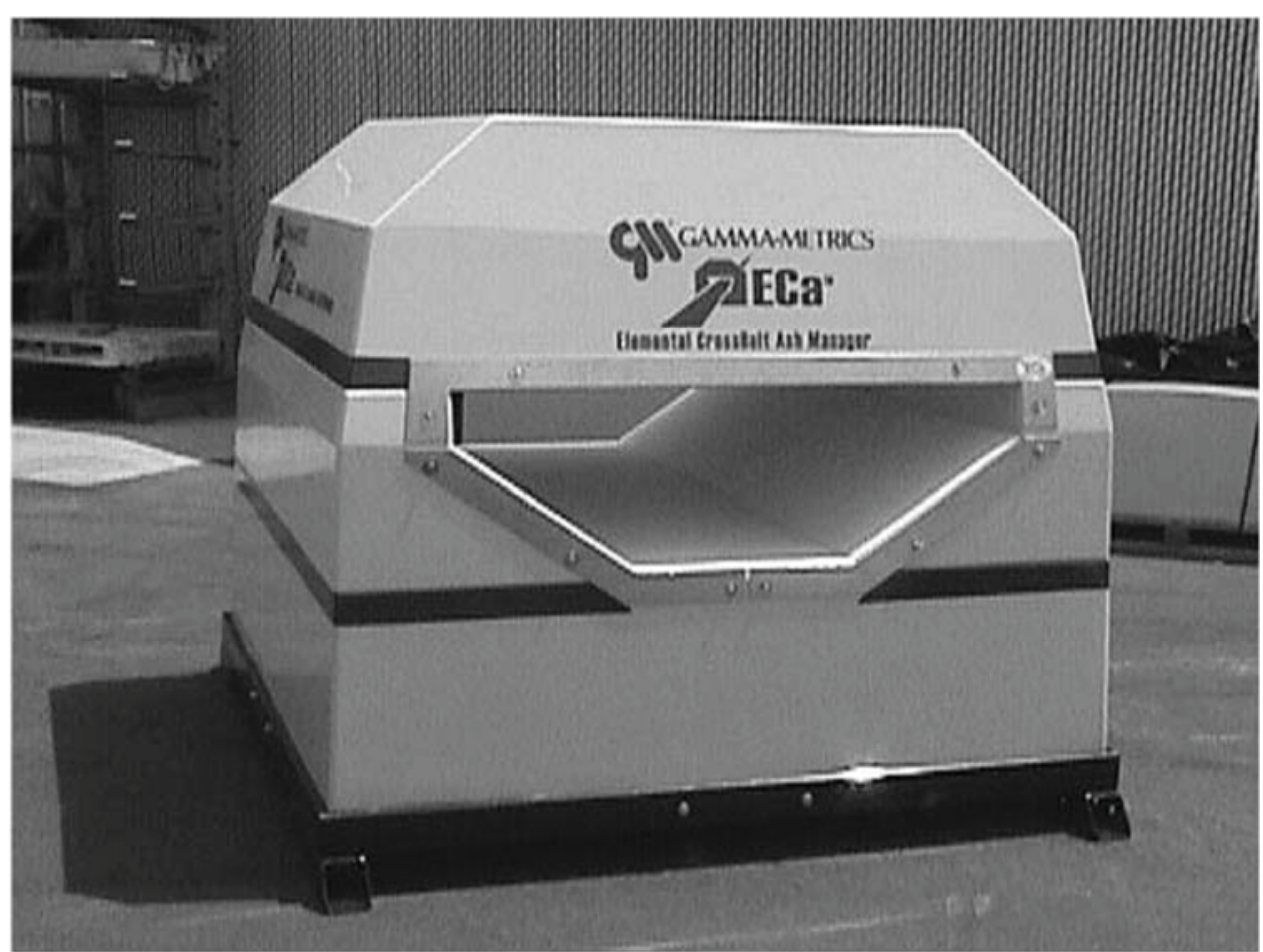

Figure 7 Photograph of an industrial PGNAA-based material analyzer [8]

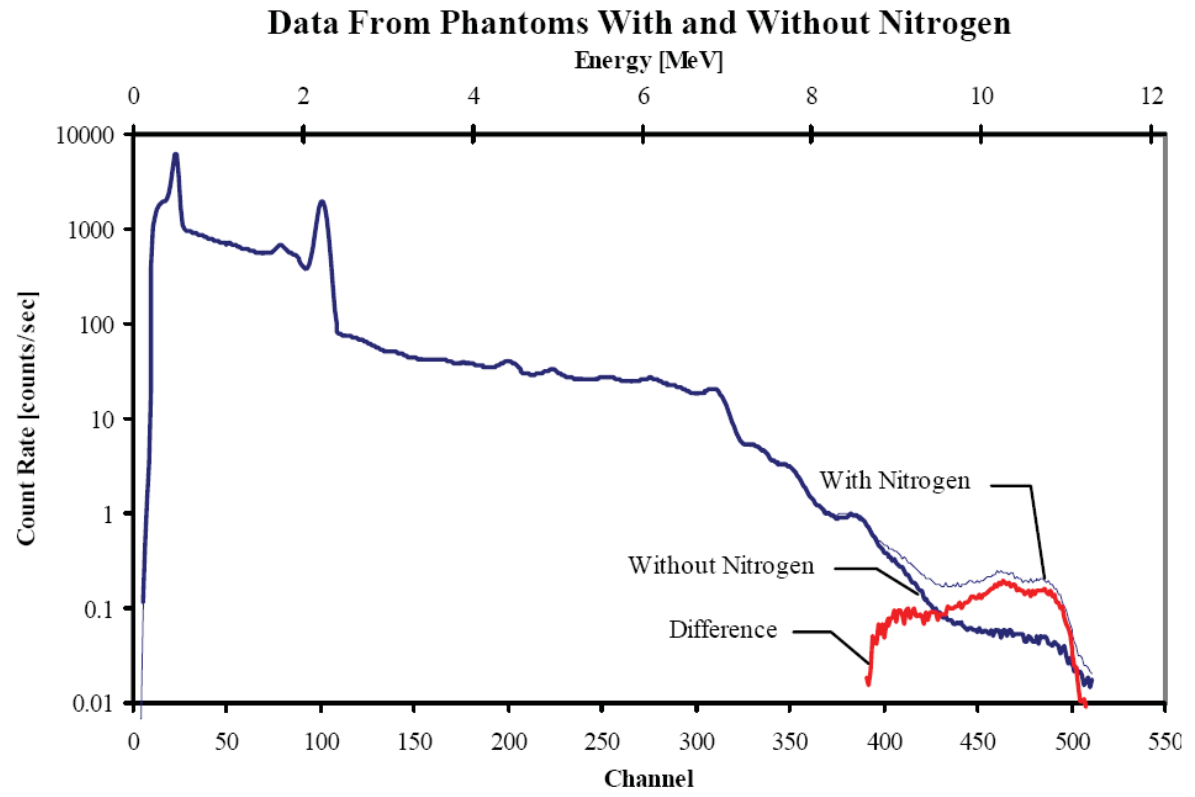

Figure 8 One-hour gamma-ray spectrum from an industrial PGNAA analyzer interrogating $19.5 \mathrm{~L}$ of liquid containing $2.7 \mathrm{wt}$. \% nitrogen. [8]

\section{Alternate Techniques for Assaying Nitric Acid}

In light of the these findings on feasibility, cost, and installed footprint, further investigations were taken to assess current industrial process monitoring technology that might be used to address the need to assay nitric acid concentrations. One traditional 
approach is the use of $\mathrm{pH}$ dip probes. ${ }^{1}$ These instruments measure the concentration of hydronium ions $\left(\mathrm{H}^{+}\right)$in a solution, using this to determine $\mathrm{pH}$ according to the relationship $\mathrm{pH}=-\log \left[\mathrm{H}^{+}\right]$. Advantages of this approach include low instrument costs, good availability, and simplicity. Drawbacks of this instrument include the need to insert the probe into the solution and the need for periodic maintenance to recharge a reference $\mathrm{pH}$ solution included in the probe. Also, the high-strength $>1 \mathrm{M}$ nitric acid range may be too acidic for these instruments to operate reliably for long periods of time. A second approach is to measure the electrical conductivity of a solution to infer molarity. This approach is used in industrial processes with strong acids but requires intrusive access for the probe to come in contact with the solution. Also, the measurements may be spoofed to read low with the addition of additives. Also, the electrical conductivity of high strength acids is not single-valued, as shown in Figure 9.[9] Maintenance for these systems is not trivial, requiring the process stream to be drained and flanges to be removed to allow the flow-through probe to be removed.

A third approach to determine the

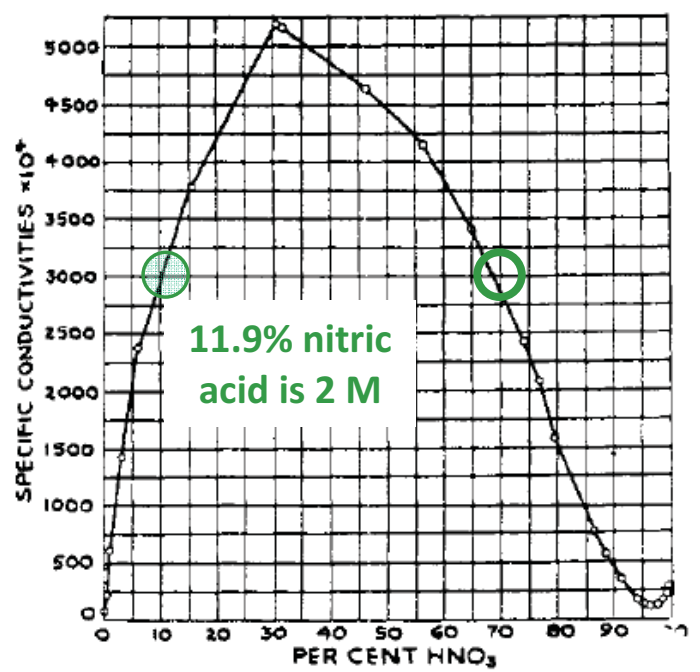

Figure 9 Electrical conductivities of nitric acid at $28^{\circ} \mathrm{C}$ [9] strength of a strong nitric acid solution is to measure the speed of sound in the solution. ${ }^{2}$ The speed of sound in fluids is related to the fluid density, and in nitric acid the solution density is related to the $\mathrm{HNO}_{3}$ concentration. These sensors incorporate ultrasonic transducers to generate sound waves in the medium and to measure return signals; when the measurement geometry is chosen to have a known flight path the transit time and sound speed can then be determined. In some instances these sensors require pipe penetrations to insert a probe. In other instances the equipment may be strapped to the outside of the pipe. According to one vendor contacted for this report this type of measurement should be able to generate measurements over the $1-4 \mathrm{M}$ range with a precision of $0.5 \%$; also, the maintenance of externally mounted devices is minimal and the expected service life of these sensors exceeds 7 years. Calibration for these sensors, as with the other approaches, must be made against laboratory benchmarks. The ultrasonic measurement technique is temperature sensitive but the devices generally incorporate a method for temperature compensation. One challenge for these systems is that pipe-wall thinning or small chemical changes could confuse the sensor readings, producing altered sound velocity values which would be misinterpreted as false acid strengths.

\footnotetext{
${ }^{1}$ For example, see the DolpHin Series pH sensors at www.invensys.co.

${ }^{2}$ For example, see the DensiCheckTX instrument from www.canongatetechnology.co.uk or the LiquiSonic instrument from www.sensotech.com.
} 


\section{Summary of Nitric Acid Assay Techniques}

A summary of different approaches for analyzing nitric acid is presented in Table 4. Each approach has strengths and weaknesses in the context of application for process monitoring and safeguards data collection within an advanced fuel cycle facility. Under specific conditions all of the approaches could potentially be used for near real-time nitric acid concentration monitoring. However, issues impacting plant operations (service, maintenance, calibration, reliability, etc.) would likely be the key factors impacting a future plant's operations and productivity, and the acceptance of this type of instrument by a facility operator. Based on this preliminary review the most likely candidate for use in an advanced reprocessing facility would be an instrument based on ultrasonic analysis to determine fluid density and subsequently $\mathrm{HNO}_{3}$ concentration. Further research in this area would include evaluations of the long term stability, accuracy, precision, and overall performance for these types of sensors. Also, work would be needed to investigate potential mechanisms for spoofing the system, the utility of these approaches, and potential countermeasures for detecting spoofing. PGNAA based analyses might also provide a viable solution for this measurement requirement. However, the complexity of installation and operation of this type of instrument greatly exceeds that of the ultrasonic based approach and would probably not be warranted following a more detailed systemlevel performance analysis. Based upon exchanges with vendors of commercial-of-theshelf (COTS) ultrasonic systems the purchase price is estimated to be $\sim \$ 7000$ per unit, not including installation costs needed to connect the sensor to a data handling system.

Table 4 Sensor techniques for assaying strong nitric acid concentrations

\begin{tabular}{|c|c|c|c|c|}
\hline Parameter & $\begin{array}{c}\text { Standard pH } \\
\text { Meter }\end{array}$ & $\begin{array}{c}\text { Electric } \\
\text { Conductivity } \\
\text { Measurement }\end{array}$ & $\begin{array}{c}\text { Ultrasonic } \\
\text { Analysis }\end{array}$ & PGNAA \\
\hline Installation Cost & Low & Low & Low & High \\
\hline $\begin{array}{c}\text { Operational/ } \\
\text { Maintenance Cost }\end{array}$ & Medium & Medium & Low & Low \\
\hline $\begin{array}{c}\text { Facility Impact } \\
\text { Including Access } \\
\text { Requirements and } \\
\text { Instrument Size }\end{array}$ & Medium & Medium & Low & Medium \\
\hline $\begin{array}{c}\text { Does Calibration } \\
\text { Require Laboratory } \\
\text { Analysis Support }\end{array}$ & Yes & Yes & Yes & Maybe \\
\hline $\begin{array}{c}\text { Can Calibration } \\
\text { Be Done Without } \\
\text { Impacting the Process } \\
\text { Stream }\end{array}$ & No & No & No & Maybe \\
\hline $\begin{array}{c}\text { Susceptibility to } \\
\text { Tampering to Produce } \\
\text { False Low- } \\
\text { Concentration Values }\end{array}$ & Medium & Medium & Medium & Low \\
\hline
\end{tabular}




\section{Ultraviolet Imaging for Detecting Alpha-Emitting Radioisotopes}

Preliminary scoping studies were carried out this year to evaluate the potential applicability for using ultraviolet (UV) imaging as a method for localizing alpha-particle induced air ionization - as a means for characterizing contamination and for detecting and quantifying hold-up within advanced fuel cycle facilities. The concept of using UV imaging to locate alpha emitting radionuclides has been demonstrated by a French research team to be a powerful tool for detecting contamination.[10] Extrapolating the concept, we believe it also shows promise as a safeguards inspection tool for detecting material hold-up in fissile material processing facilities, for verifying the non-operational status of work spaces at declared facilities, and for detecting undeclared work activities. A related technology based on sensing the electric charge from air ionization has been developed at Los Alamos National Laboratory, strengthening the potential nonproliferation and counterproliferation value for methods detecting alpha-particle induced air ionization.[11]

For this work a high-performance scientific imaging camera capable of acquiring data in the UV range was borrowed and used to conduct proof-of principle tests. Experiments were carried out at INL's Health Physics Instrumentation Laboratory. An alpha-emitting plutonium check source comprised of a metal disk to which ${ }^{239} \mathrm{Pu}$ had been electrodeposited was used to induce air ionization. The plutonium deposited on this source was very thin and the emitted alpha spectrum demonstrated little degradation as a result of self-attenuation. The source strength was approximately 2.6 million disintegrations per minute. A photograph of the source is shown

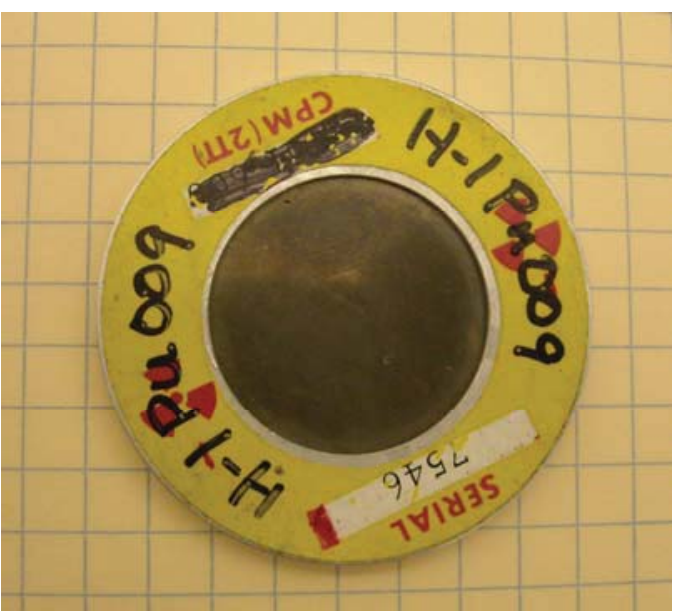

Figure 10 The ${ }^{239} \mathrm{Pu}$ alpha source used for UV imaging tests at HPIL in Figure 10. The source was mounted $60-\mathrm{cm}$ from the charge coupled device (CCD) image plane of the camera. Three measurements were taken, one short-exposure picture in visible light to see the set-up and two 50minute exposures to capture the weak UV image. (The two long duration exposures were combined in software, allowing for the removal of cosmic-ray induced artifacts generated in the CCD imager during the long integration periods.) It was necessary to perform the long duration measurements in darkness in order to capture the UV signal absent the visible light background. Photographs of the physical setup used during data collection are shown in Figure 11 and Figure 12. 


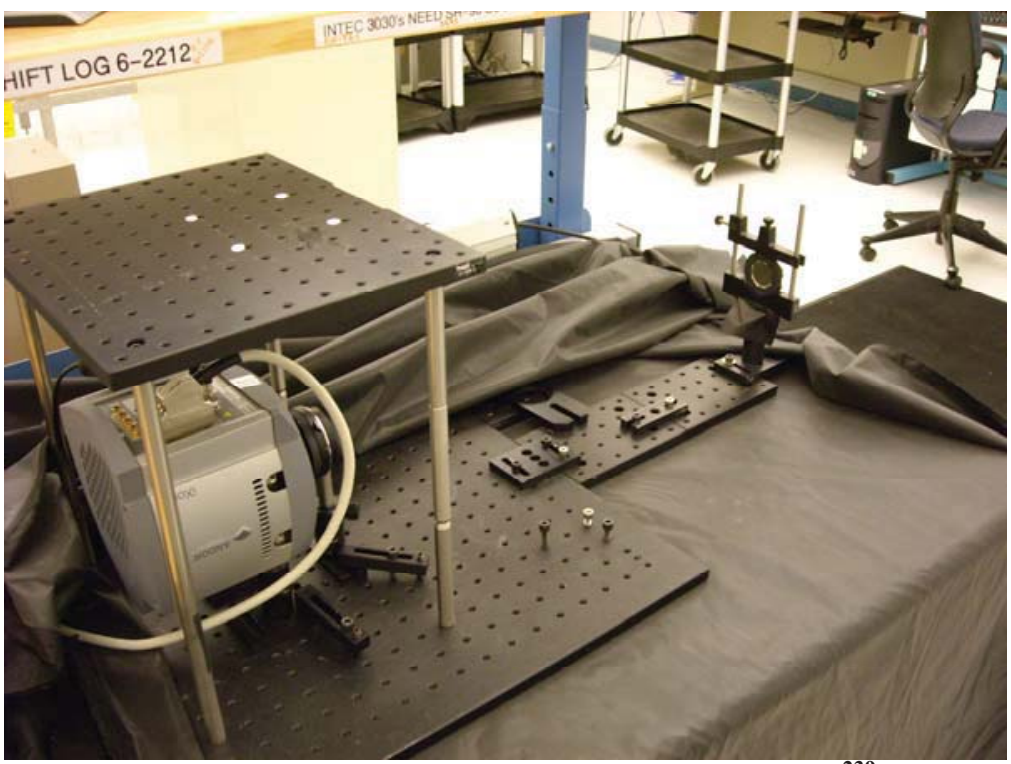

Figure 11 Photograph of the apparatus showing the camera (left) and ${ }^{239}$ Pu source (right), during measurements the black cloth was draped over the setup. In some cases a black pipe was attached to the end of the camera and the source was placed inside the pipe, rather than using the mounting bracket shown here.

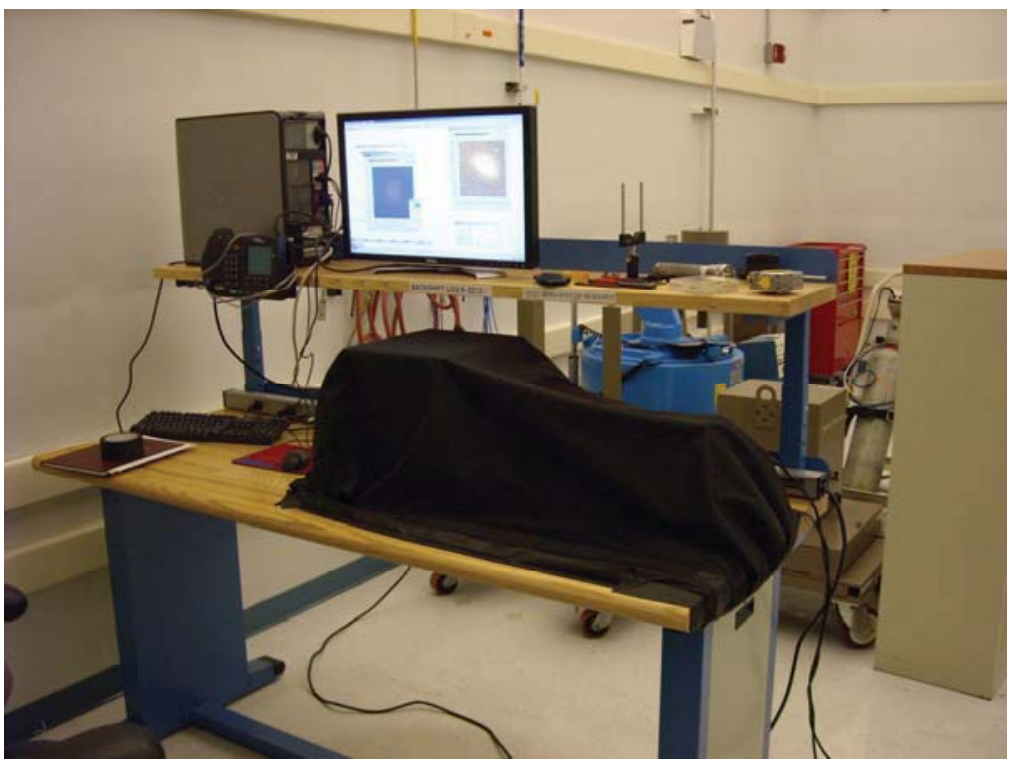

Figure 12 Photograph of the setup used to collect the UV images, showing the black cloth draped over the apparatus

Data from these measurements are shown separately in Figure 13 and Figure 14. The UV air excitation is clearly visible above the alpha-emitting surface of the check source. For these preliminary tests a low-quality imaging lens was used, for future work a better quality UV lens will be used. Additionally, we plan to examine the use or UV optical notch filters to test the feasibility of acquiring images under ambient light conditions. During the next phase of this work we will also begin modeling work to simulate alpha energy deposition in air and in inert atmosphere environments such as nitrogen and argon, gasses that are often used in glove boxes and hot cells. 


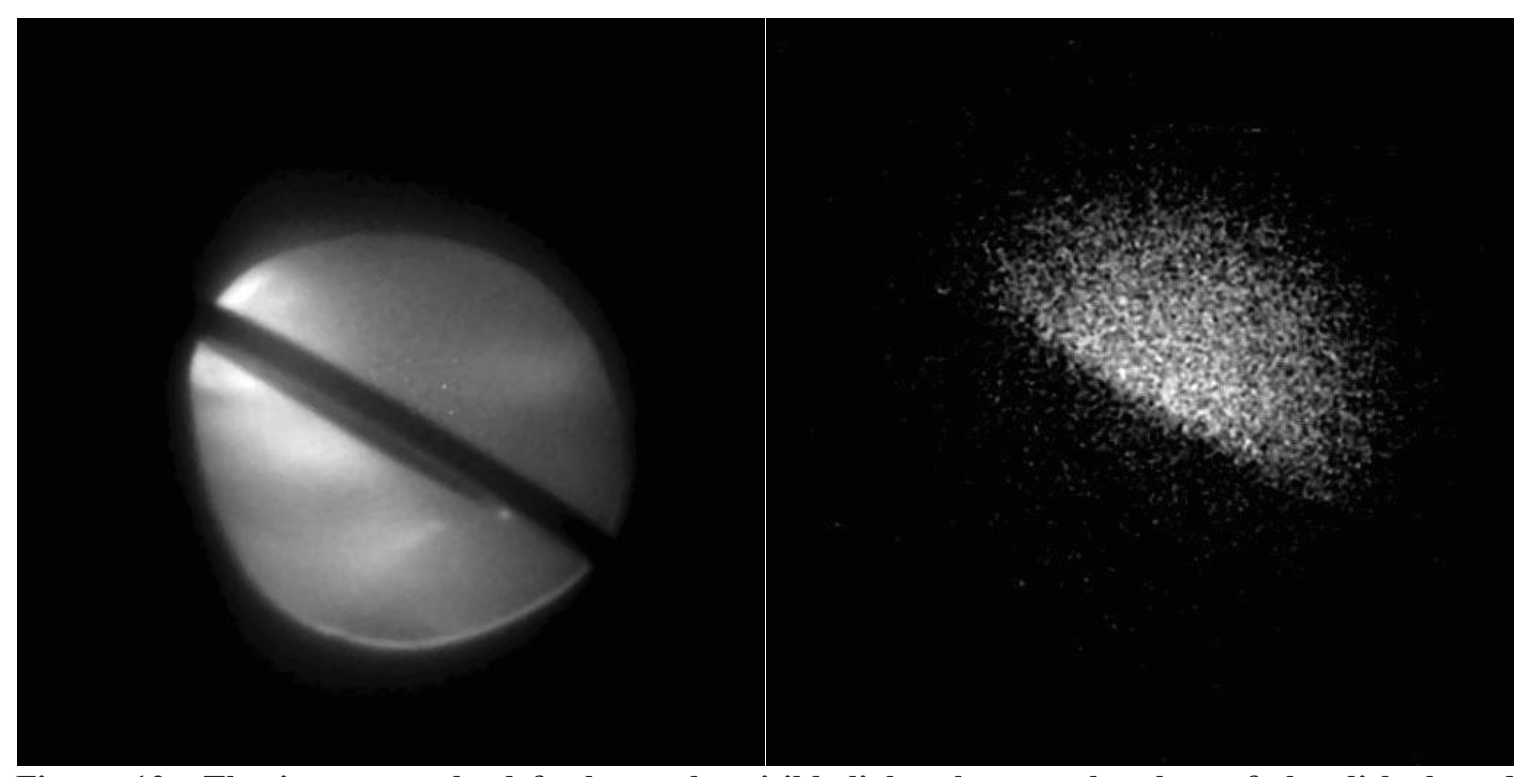

Figure 13 The image on the left shows the visible-light photograph taken of the disk-shaped plutonium check source from Figure 10 mounted at an angle at the end of a dark tube, the alpha active surface was on the upper side of the source, facing to the right.. The image on the right shows the summed data collected over two 50-minute collection periods with the source in a dark environment, corresponding to the UV signal generated in the air above the source.

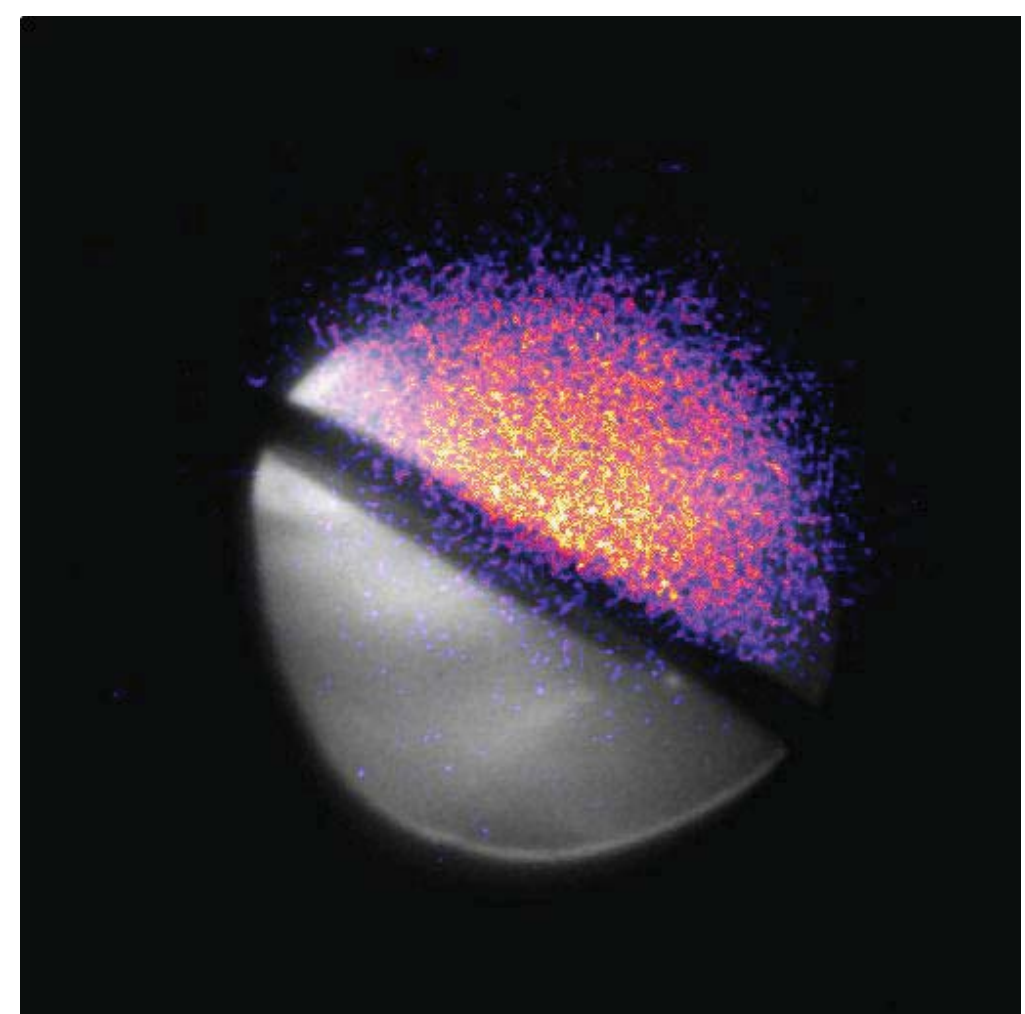

Figure 14 Composite image of the views in Figure 13; the UV light produced by air-ionization is presented in color while the visible light image of the source remains in grayscale. 


\section{Summary}

During FY-2009 four small-scale projects have been conducted at INL as part of the MPACT program: 1) MOX Fuel Modeling, Simulations, \& Experiments (with the University of Michigan); 2) Fast-Neutron Coded -Aperture Imaging (with ORNL); 3) Review of On-Line Monitoring Techniques to Determine Cold-Stream Nitric Acid Strength, and 4) Ultraviolet Imaging for Detecting Alpha-Emitting Radioisotopes. These tasks address several different areas that are important in the context of future technologies for domestic nuclear safeguards. One theme within these projects is the need to explore different safeguard concepts for dealing with MOX fuel. Advanced MOX fuel is one focus of study within the Fuel Cycle Research and Develop program, new approaches will be needed to protect and secure this material within fuel manufacturing and reprocessing facilities. A second theme within these projects is the need to develop next-generation tools for detecting, characterizing, and quantifying fissile material hold-up in material processing facilities. Dealing with, and accounting for, material hold-up presents a significant burden on fissile material processing facilities, methods for improving and streamlining this process will be a great benefit for future domestic fuel reprocessing and production facilities. A third theme of this work is the consideration of non-contact, nondestructive, stand-off assay techniques versus destructive or close-contact approaches. For material protection, accounting, and control non-contact methods provide a significant advantage over alternate technologies; the development of technologies satisfying this requirement have the greatest potential benefit for future domestic facilities requiring safeguards.

Important aspects of long-term, science-based research and development (R\&D) include small-scale experiments, theory development, and advanced modeling and simulation with validation experiments; the projects described here embrace the paradigm for the "science-based" R\&D approach. For example, the MOX fuel analysis work has used small-scale experiments at the University of Michigan to prove concepts and develop ideas for how to collect and process data. Simultaneously, simulation and modeling studies have been used to understand the results of these experiments and to guide the development and planning of the project. Combining this information new theories are under consideration for how to extract increasing amounts of data from the limited but complex data streams associated with the time-correlated neutron and gamma-ray data from the liquid scintillators. Finally, validation experiments have been carried out to benchmark our modeling efforts and provide confidence moving forward that simulations of these techniques are providing accurate results.

The project team presented one conference paper on MOX fuel simulation and modeling in July at the $50^{\text {th }}$ Annual Meeting of the Institute of Nuclear Materials Management (INMM) in Tucson Az.; this presentation won a prestigious award for team member Jennifer Dolan, who delivered the talk, in the student-presentation category. The talk from the INMM meeting has been selected for publication in the peer-reviewed Journal of Nuclear Material Management. A second paper focusing on the results from the June experiments is currently being prepared and will be presented at the upcoming Institute of Electrical and Electronics Engineers Nuclear Science Symposium in October of this year. A third paper dealing with the alpha-imaging technique is currently under development and will be submitted for publication in the Proceedings of SPIE next spring. A fourth paper detailing the July experiments with ORNL is under consideration 
but has not yet been developed. In 2010 we plan to present a follow-on paper at the INMM meeting to review the June validation experiments conducted to support the MOX fuel analysis simulation and modeling.

Looking forward to FY-2010 we plan to continue working to explore methods for using liquid scintillator instrumentation and how they may best be used for assaying and fuel cycle materials, particularly for characterizing MOX fuel. Building upon the experiments and validation work in FY-2009, new simulation and modeling efforts will be expanded to begin addressing active interrogation measurements. Following smallscale tests and modeling through out the winter of 2010 a second set of validation experiments is planned for the summer of 2010. During FY-2010 the ORNL team has indicated interest in updating their sensor systems from plastic scintillators to liquid scintillators; INL will support this activity as needed. Reflecting the recent shift to emphasize exploratory work rather than engineering analyses within the Fuel Cycle Research and Development program no further work is anticipated in the area of coldstream nitric acid concentration determination, although this work could be expanded upon in the future if needed. Building upon FY-2009 successes in the area of airionization alpha radiation detection we will continue to explore this measurement technique as a tool to support next-generation domestic safeguard activities and fissile material hold-up detection. In the past INL has maintained an informal information exchange with safeguards researchers at Lawrence Berkeley National Laboratory, this activity will continue and if concrete opportunities for collaboration are identified they will be pursued.

\section{Acknowledgement}

The authors would like to acknowledge and thank the staff at INL's Materials and Fuels Complex, the ZPPR facility, and the HPIL facility for their support and enthusiasm for the experiments described in this report. The work in this report was sponsored by the U.S. Department of Energy's Fuel Cycle Research and Development program and its Materials Protection, Accounting, and Control for Transmutation (MPACT) campaign. Additional support was also provided by INL in the form of Laboratory Directed Research and Development funds from INL's Nuclear Nonproliferation Initiative.

\section{Reference}

1 Dolan, J. L., Flaska, M., Pozzi, S. A., and Chichester, D. L., " Measurement and Characterization of Nuclear Material at Idaho National Laboratory," Report INL/CON-09-16103, Idaho National Laboratory, Idaho Falls, Idaho (2009).

2 Chichester, D. L., Pozzi, S. A., Dolan, J. L., Flaska, M., Johnson, J. T., Seabury, E. H., and Gantz, E. M., "Neutron Emission Characteristics of Two Mixed-Oxide Fuels: Simulations and Initial Experiments," Report INL/EXT-09-16566, Idaho National Laboratory, Idaho Falls, Idaho (2009).

3 Pozzi, S. A., Padovani, E., and Marseguerra, M., "MCNP-PoliMi: A Monte Carlo Code for Correlation Measurements," Nucl. Inst. Meth. Phys. Res. A 513 (2003) 550558. 
4 Chichester, D. L., Seabury, E. H., Turnage, J. A., Brush, B. A., and Perry, E. F., "Capabilities of the INL ZPPR to Support Active Interrogation Research with SNM," AIP Conf. Proc. 1099 (2009) 647-651.

5 Klann, R. T. Austin, B. D., Aumeier, S. E., and Olsen, D. N., "Inventory of Special Nuclear Materials from the Zero Power Physics Reactor," Report ANL-NT-176, Argonne National Laboratory-West, Idaho Falls, Idaho (2001).

6 Regalbutto, M., Bakel, A., Pereira, C. Krebs, J., and Hebden, A., "Update on Process Monitoring Task," Argonne National Laboratory, GNEP Safeguards Working Group Meeting, Gaithersburg, Md., June 17-18 (2008).

7 Caffrey, A. J., Cole, J. D., Gehrke, R. J., and Greenwood, R. C., "Chemical Warfare Agent and High Explosive Identification by Spectroscopy of Neutron-Induced Gamma Rays," IEEE Trans. Nucl. Sci. 39 (1992) 1422-1426.

8 Chichester, D. L. and Empey, E., "Measurement of Nitrogen in the Body Using a Commercial PGNAA System - Phantom Experiments," App. Rad. Iso. 60 (2004) 5561.

9 Coster, H. M., "Application of Conductivity Measurement of Nitric Acid Concentration to Plant Control," Ind. Eng. Chem. 25 (1933) 980-982.

10 Lamadie, F., Delmas, F., Mahe, C., Girones, P., Le Goaller, C., and Costes, J. R., "Remote Alpha Imaging in Nuclear Installations: New Results and Prospects," IEEE Trans. Nucl. Sci. 52 (2005) 3035-3039.

11 Koster, J. E., Johnson, J. P., MacArthur, D. W., and Walters, S. G., "Alpha Detection as a Probe for Counter Proliferation," Report LA-UR-94-1946, Los Alamos National Laboratory, Los Alamos, N.M. (1994). 